\title{
Betreuen und pflegen: Ein Schlusswort
}

von Prof. Annemarie Kesselring

Die Gesellschaft steht der Pflege und Betreuung von Menschen, die auf die Unterstützung anderer angewiesen sind, mit gemischten Gefühlen gegenüber. Da gibt es einerseits das noble «Florence-Nightingale»-Bild, in dem die Verdienste der Pflegenden bewundert und als etwas Aussergewöhnliches, das man sich selbst nie zutrauen würde, dargestellt werden. Das Gegenbild stellt Pflegen als wenig heroische Tätigkeit dar, die nur geringe Ausbildung braucht, weil sie - wie das Erziehen von Kindern - als etwas Selbstverständliches und Gewöhnliches betrachtet wird. Diese gewöhnlichen und manchmal auch als schmutzig empfundenen Arbeiten sind zwar für das Wohl der Gemeinschaft notwendig, die Menschen - meistens Frauen -, die sie vollbringen, geniessen jedoch wenig gesellschaftliche Anerkennung.

Bei Demenzbetroffenen ist diese Anerkennung auch im Gesundheitswesen und sogar unter den Pflegenden selbst gering, weil die medizinische Wertehierarchie klar ist: Anerkennung findet, wer Heilung ermöglicht. In dieser Wertehierarchie nimmt die Betreuung und Pflege Demenzbetroffener einen niedrigen Platz ein, weil sie vielerorts als einfache Versorgungsarbeit verstanden wird, die kaum zur «Besserung» beiträgt.

Auch in medizinisch-pflegerischen Berufskreisen ist das Verständnis, dass es sich bei der Pflege Demenzerkrankter um eine hoch spezialisierte und äusserst anspruchsvolle Aufgabe handelt, wenig verbreitet. Es braucht Bücher wie dieses und vor allem die Erfahrungen, die ihm zugrunde liegen, damit die 
hier vorgestellten Betreuungsansätze und Organisationsformen in breiten Kreisen Beachtung finden.

Demenzbetroffene Menschen leben in sehr unterschiedlichen Welten, die in diesem Handbuch so nachvollziehbar wie möglich beschrieben werden. Keine dieser Welten kann von den Pflegenden auf Grund eigener Erfahrung wirklich nachvollzogen werden. Somit ist klar, dass die Betreuenden von demenzbetroffenen Menschen stets Lernende, Suchende und Entdeckende sind, Menschen mit viel Einfühlungsgabe und Phantasie, die sich an die Befindlichkeit der Betroffenen herantasten. Es gilt, die Betroffenen immer besser kennen zu lernen, zu spüren, wer sie waren und wer sie heute sind und vor allem, wie man mit ihnen nicht nur durch Worte kommunizieren kann.

Die Pflege von Menschen, die an Demenz leiden, erfordert deshalb ständig, einen grossen Teil dessen zu hinterfragen, was für Menschen mit intakten geistigen Fähigkeiten als völlig selbstverständlich gelebt wird. Pflegende, welche die Kunst dieser Pflege leben, gleichen eher Weggefährten auf einer Reise durch eine völlig veränderte und wenig bekannte Alltagswelt. Allerdings muss eine solche Reisebegleitung gelernt und in neuen Situationen immer wieder geübt und auf den zu begleitenden Menschen angepasst werden.

In Nordschweden wurde in den vergangenen 30 Jahren die Pflege von demenzbetroffenen Menschen mittels vieler Studien erforscht. Die Forschungsergebnisse flossen als Grundlage in die Aus- und Weiterbildung des Pflegepersonals, aber auch in die Arbeitsorganisation und Konzeption des Pflegealltags ein. Durch die Teilnahme an den Studien und Schulungen wurde breites Interesse geweckt, Pflege wurde plötzlich spannend. Die Pflegenden lernten, die Situation des Betroffenen differenziert zu erfassen, um daraus abzuleiten, welche Fähigkeiten des betreuten Menschen sie erhalten und fördern und wo 
und wie sie die Menschen in einer Art und Weise unterstützen konnten, die ihr Selbstwertgefühl nicht verletzte. Sie lernten, was zu tun war, wenn fast alle Aktivitäten des täglichen Lebens übernommen werden mussten, und begannen sich dafür zu interessieren, wie sie Kontakt zu Demenzbetroffenen herstellen konnten. Durch die Aufmerksamkeit, die den Patienten und den Pflegenden, der Pflege und ihrer Organisation durch Forschung und Lehre zukam, war die Pflege von schwerst abhängigen Menschen auf einmal so interessant geworden, dass Pflegende nicht mehr auf der Medizin oder Chirurgie eine Stelle suchten, sondern in Institutionen der Langzeitpflege, in denen geforscht und gelehrt wurde [A. Norberg, persönliches Gespräch].

Auch in der Schweiz nimmt die Zahl der Pflegenden zu, die sich in das Spezialgebiet der Pflege Demenzbetroffener vertiefen. Noch sind es wenige, aber ihre Stimme wird stärker und ihr Einfluss auf die Pflege in den Institutionen nimmt zu. Damit jedoch Veränderungen, wie sie in diesem ausgezeichneten Handbuch beschrieben sind, selbstverständliche Realität werden, braucht es noch viel Arbeit im Bereich der Organisationsentwicklung und des innerbetrieblichen Lernens.

Wichtige Fragen müssen von den Pflegenden immer wieder gestellt werden, zum Beispiel: "Was tun wir hier, dass alle so zufrieden sind? Was ist hier problematisch? Was ist geschehen, dass sich die Situation so und so entwickelt hat? Was haben wir zur Entwicklung der Situation beigetragen bzw. unterlassen? Wie können wir es anders angehen?» Kompetent geleitete, betriebsinterne Fallstudien erlauben eine hohe Identifikation der Lernenden mit der Situation und ermöglichen es, Problemlösungen gemeinsam anzugehen, zu evaluieren und allenfalls weiterzuentwickeln. Betriebsinternes Lernen fördert die Qualitätsentwicklung und interdisziplinäre Zusammenarbeit. 
Im vorliegenden Handbuch finden sich zahlreiche Impulse für einen Kulturwandel in der Pflege- und Heimwelt. Für die Betreuung und Pflege von Demenzbetroffenen werden echte Alternativen aufgezeigt und Grundlagen für eine Pflege- und Betreuungskultur geschaffen, die auf fachlicher Expertise und menschlichem Engagement gründen.

Annemarie Kesselring

Institut für Pflegewissenschaft

Universität Basel 